\title{
Epigenetic silencing of KAZALD1 confers a better prognosis and is associated with malignant transformation/progression in glioma
}

\author{
HONGJUN WANG ${ }^{1,5^{*}}$, YING FENG $^{2 *}$, ZHAOSHI BAO ${ }^{3-5^{*}}$, CHUANLU JIANG $^{1,5}$, \\ WEI YAN ${ }^{3-5}$, YONGZHI WANG ${ }^{3-5}$, CHUANBAO ZHANG $^{3-5}$, YANWEI LIU ${ }^{3-5}$, \\ QUANGENG ZHANG $^{2}$, WEI ZHANG ${ }^{3-5}$ and CHUANLU JIANG ${ }^{1,5}$
}

\footnotetext{
${ }^{1}$ Department of Neurosurgery, The Second Affiliated Hospital of Harbin Medical University, Harbin, Heilongjiang 150086;

${ }^{2}$ Department of Immunology, Insistute of Basic Medical Sciences, Capital Medical University, Beijing 100069;

${ }^{3}$ Beijing Neurosurgical Institute, Beijing 100050; ${ }^{4}$ Department of Neurosurgery, Beijing Tiantan Hospital, Capital Medical University, Beijing 100050, P.R. China; ${ }^{5}$ Chinese Glioma Cooperative Group (CGCG)
}

Received May 29, 2013; Accepted July 2, 2013

DOI: $10.3892 /$ or.2013.2706

\begin{abstract}
In order to more thoroughly analyze aberrant DNA methylation in glioma, we applied a large cohort methylation microarray including 119 glioma samples. Six genes, ADCY1, KAZALD1, KLF4, SLMAP, TETRAN and TP53INP1, were screened out through significance analysis of microarray (SAM), survival Cox-regression and certain other pre-set conditions. We focused on the KAZALD1 oncogene. KAZALD1, also known as IGFBP-rP10, belongs to the IGFBP family. We found that KAZALD1 was hypomethylated in highgrade glioma (anaplastic gliomas and glioblastomas) compared to low-grade glioma (astrocytoma, oligodendrocytoma and oligoastrocytoma) using methylation microarrays $(\mathrm{P}<0.001)$. Immunohistochemistry (IHC) of 91 glioma samples showed that the KAZALD1 expression scores of high-grade glioma samples were higher compared to the scores of low-grade gliomas $(\mathrm{P}<0.001)$. In high-grade gliomas, overall survival (OS) was shorter for patients with KAZALD1 hypomethylation or overexpression compared to those without. Decreased KAZALD1 expression in glioma inhibited cell proliferation and invasion both in vitro and in vivo. On the basis of these observations and the results from subset analysis, it is reason-
\end{abstract}

Correspondence to: Professor Chuanlu Jiang, Department of Neurosurgery, The Second Affiliated Hospital of Harbin Medical University, 246 Xuefu Road, Harbin, Heilongjiang 150086 , P.R. China

E-mail: jc16688@163.com

Dr Wei Zhang, Department of Neurosurgery, Beijing Tiantan Hospital, Capital Medical University, Beijing 100050, P.R. China

E-mail: zhangwei_vincent@yahoo.com.cn

*Contributed equally

Key words: hypomethylation, KAZALD1, glioma, prognosis able to conclude that KAZALD1 promoter hypomethylation is an important prognostic biomarker in glioma. KAZALD1 promotes glioma malignant progression through invasion and proliferation.

\section{Introduction}

Gliomas are the most common and most aggressive types of primary brain tumor in humans. Despite recent advances in treatment by a combination of surgery and chemotherapy and/ or radiotherapy, the prognosis of malignant glioma remains extremely poor. Glioblastoma (GBM) is the most common and malignant glioma, and has a median survival time of approximately one year (1-7). In the United States, $75 \%$ of glioma patients die within 5 years of diagnosis (8). There is, therefore, an urgent need for the development of a prognostic biomarker for this disease.

To date, a considerable amount of research has discovered that epigenetic mechanisms play an important role in tumors. Aberrant epigenetic mechanisms, such as promoter hyper/hypomethylation, histone modifications, or non-coding RNA expression, are key in tumor formation (9). Moreover, DNA methylation is typically a more stable and inheritable epigenetic pattern that can persist for several cell generations, which potentially broadens its practical applicability in the clinic (10). Changes in DNA methylation patterns are an important hallmark of tumor development and progression (11). Although the role of hypermethylation in the silencing of tumor suppressor genes is now well-documented (12), a reduction in the level of methylation also contributes to neoplastic progression in numerous types of human cancer, including gliomas $(13,14)$.

KAZALD1 is also known as IGFBP-rP10, BONO1, FKSG28 or FKSG40. This gene encodes a secreted member of the insulin growth factor-binding protein (IGFBP) superfamily. It contains an $\mathrm{N}$-terminal insulin growth factor-binding domain, a central Kazal-type serine protease 
inhibitor and follistatin-like domain, and a C-terminal immunoglobulin-like domain. Studies of the mouse ortholog suggest that this gene product may have a function in bone development and bone regeneration. The insulin-like factor binding domain has high affinity for insulin-like growth factors, while the Kazal domain contains sequence and structural homology to serine proteases and follistatins (15). The immunoglobulin-like domain appears to have a molecular binding function perhaps regulating cell adhesion and extracellular ligand binding (16), and is associated with regions of matrix mineralization in bone and dentin matrices (17). Invasion and proliferation may be the way KAZALD1 promotes tumor progression.

In the present study, promoter methylation status of KAZALD1 and its correlation with clinicopathological parameters were evaluated in our methylation microarray and in 91 independent samples. The promoter methylation status of KAZALD1 in glioma was investigated to determine any correlation between tumors and DNA methylation. Correlation of KAZALD1 methylation status with clinical data was investigated to assess its predictive and prognostic value for glioma patients. Expression of KAZALD1 in these tumors was also assessed to determine whether promoter hypomethylation correlated with protein overexpression in gliomas. Regular follow-up of these patients and correlation of molecular findings with disease outcome emphasized the prognostic relevance of KAZALD1 methylation and expression in glioma patients. Functional assays in glioma cell lines were also performed.

\section{Materials and methods}

Patients and samples. All patients were from the Chinese Glioma Genome Atlas (CGGA). The patients selected in the present study underwent surgical resection between January 2006 and December 2010 and subsequently received concomitant and adjuvant temozolomide and radiotherapy. Clinical data, including patient age at diagnosis, gender and preoperative Karnofsky performance status (KPS) score were obtained from the medical records. Overall survival (OS) time, defined as the period from operation to mortality, was calculated mainly when patients visited the clinics or by phone interview with patients and/or their relatives. Patients who succumbed to non-primary diseases were excluded (18). Tumor tissue samples were obtained by surgical resection before the treatment with radiation and chemotherapy. Respective specimens were snap-frozen and stored in liquid nitrogen until nucleic acid extraction. The present study was approved by the Ethics Committee of the Capital Medical University, and written informed consent was obtained from all patients. Only samples with $>80 \%$ tumor cells were selected.

DNA extraction. All tissue samples were immediately snapfrozen in liquid nitrogen after surgery. A hematoxylin and eosin-stained frozen section was prepared for assessment of the percentage of tumor cells before DNA extraction. Genomic DNA was isolated from frozen tumor tissues using the QIAamp DNA Mini kit (Qiagen) according to the manufacturer's protocol. DNA concentration and quality were measured using the NanoDrop ND-1000 spectrophotometer (NanoDrop Technologies, Houston, TX, USA).

Genome-wide DNA methylation profiling. A series of 119 glioma samples (63 low-grade gliomas, 33 anaplastic gliomas and 23 glioblastomas) were measured by methylation microarray. We used the Illumina Infinium Human Methylation 27 BeadChips (Illumina Inc.), as previously described (19). The BeadChip contains 27,578 highly informative $\mathrm{CpG}$ sites covering more than 14,000 human RefSeq genes. This allows researchers to interrogate all these sites per sample at a single nucleotide resolution. Bisulfite modification of DNA, chip processing and data analysis were performed following the manufacturer's manual at the Wellcome Trust Centre for Human Genetics Genomics Laboratory (Oxford, UK). The array results were analyzed with the BeadStudio software (Illumina).

Immunohistochemistry. Immunohistochemistry (IHC) was performed as previously described (20). Briefly, surgical biopsies from the patients above were fixed in formalin, routinely processed and paraffin-embedded. Five micronthick sections were prepared, and immunohistochemical staining with streptavidin-biotin immunoperoxidase assay was performed using goat polyclonal antibody to KAZALD1 (Santa Cruz Biotechnology). The degree of immunostaining of sections was viewed and scored separately by two independent investigators. The scores were determined by combining the proportion of positively stained tumor cells and the intensity of staining. The proportion of positively stained tumor cells was graded as follows: 0 , no positive tumor cells; $1,<5 \%$ positive tumor cells; $2,5-20 \%$ positive tumor cells; and 3, $>20 \%$ positive tumor cells (5). The intensity of staining was recorded on a scale of 0 (no staining), 1 (weak staining, light yellow), 2 (moderate staining, yellowish brown) and 3 (strong staining, brown). The staining index was calculated as follows: staining index $=$ staining intensity $\mathrm{x}$ proportion of positively stained tumor cells. High KAZALD1 expression was defined as a staining index score $>6$, while low expression was defined as a staining index $\leq 6$.

Cell lines and cell culture. The human glioblastoma cell lines LN229 and U251 were obtained from the Institute of Biochemistry and Cell Biology, Chinese Academy of Science, Shanghai, China. The cells were maintained in Dulbecco's modified Eagle's medium (DMEM; Gibco, Carlsbad, CA, USA) supplemented with $10 \%$ fetal bovine serum (FBS) and incubated at $37^{\circ} \mathrm{C}$ in $5 \% \mathrm{CO}_{2}$. Upon $80 \%$ confluency, cells were starved in DMEM with $1 \%$ FBS for $24 \mathrm{~h}$ and maintained in this low-serum condition for the course of the treatment.

KAZALDl gene knockdown by siRNA. Specific oligos targeting the KAZALD1 gene were selected (OriGene Technologies, Inc., Rockville, MD, USA). siRNA1, the most efficient one, screened from three siRNAs, was used to knockdown KAZALD1 (sequence, 5'-GCUAGGCACCAAUAAAC AUUUCUAA-3'). Logarithmically growing cells were seeded at a density of $10^{5}$ cells per $6-\mathrm{cm}$ dish and transfected with $5 \mu$ mol KAZALD1 siRNA using Lipofectamine ${ }^{\circledR} 2000$ (Invitrogen), according to the manufacturer's instructions. 
Forty-eight hours later, cells were used for in vitro functional assays as described below.

Western blot analysis. Following the cell treatment, cell lysates were prepared via lysis buffer, electrophoresed onto SDS-polyacrylamide gels and transferred to polyvinylidene difluoride membranes. Membranes were probed with rabbit antibodies against KAZALD1 (Santa Cruz Biotechnology) and glyceraldehyde-3-phosphate dehydrogenase (GAPDH) (A-3; Santa Cruz Biotechnology) at dilutions of 1:1,000. Blots were detected with horseradish peroxidase-labeled anti-goat antibodies (1:5,000 dilution), developed using enhanced chemiluminescence (ECL) reagents (Amersham Pharmacia, Buckinghamshire, UK).

Transwell invasion assay. The transwell invasion assay was carried out in 24-well cell culture chambers using transwell inserts (Corning Life Sciences, Corning, NY, USA) with $8 \mathrm{~m}$ pore membrane precoated with matrigel (BD Biosciense, San Jose, CA, USA). LN229 and U251 cells were plated at the density of $1 \times 10^{4}$ per upper well in $200 \mu \mathrm{l}$ culture medium (DMEM, no FBS), control group and siRNA group, respectively. The lower chamber was filled with $500 \mu \mathrm{l}$ medium (DMEM, 12\% FBS). The cells were allowed to invade for $24 \mathrm{~h}$, after which time the non-invading cells with Matrigel matrix were removed from the upper surface of the membrane by scrubbing with a cotton-tipped swab. Cells on the lower surface of the filter were fixed for $30 \mathrm{~min}$ in methanol and glacial acetic acid mixture (3:1), air-dried briefly and stained with crystal violet. The mean number of invaded cells was counted from five preselected microscopic fields at x 200 magnification. All experiments were performed in triplicate.

Colony formation assay. LN229 and U251 cells were transfected with siRNA or a negative control for $48 \mathrm{~h}$ and were then plated into six orifice plates (1,000 per orifice) and transfected with siRNA one more time on the 6th day. On the 12th day, plates were washed with PBS and stained with crystal violet. The number of colonies with $>30$ cells was counted. The colonies were counted manually using a microscope.

Nude mouse tumor xenograft model and siRNA of KAZALDI treatment. U251 glioma cells were subcutaneously injected into 5-week-old female nude mice (Cancer Institute of the Chinese Academy of Medical Science). When the tumor volume reached $50 \mathrm{~mm}^{3}$, mice were randomly divided into two groups (six mice per group). Each group was treated with siRNA of KAZALD1 or negative control oligo in $10 \mu$ l Lipofectamine through local injection of the xenograft tumor at multiple sites. The treatment was performed once every 3 days for 15 days. The tumor volume was measured with a caliper twice a week, using the following formula: volume $=$ length $\mathrm{x}$ width ${ }^{2} / 2$.

Statistical analysis. Significance analysis of microarrays (SAM) was used for genes significantly differently methylated between high-grade and low-grade gliomas. Cox-regression was performed using Matleb software. A two-sided $\chi^{2}$ test was performed using SPSS 13.0. Kaplan-Meier survival curves were obtained and differences in the OS were tested using the log-rank test (GraphPad Prism 5). Differences of tumor cell

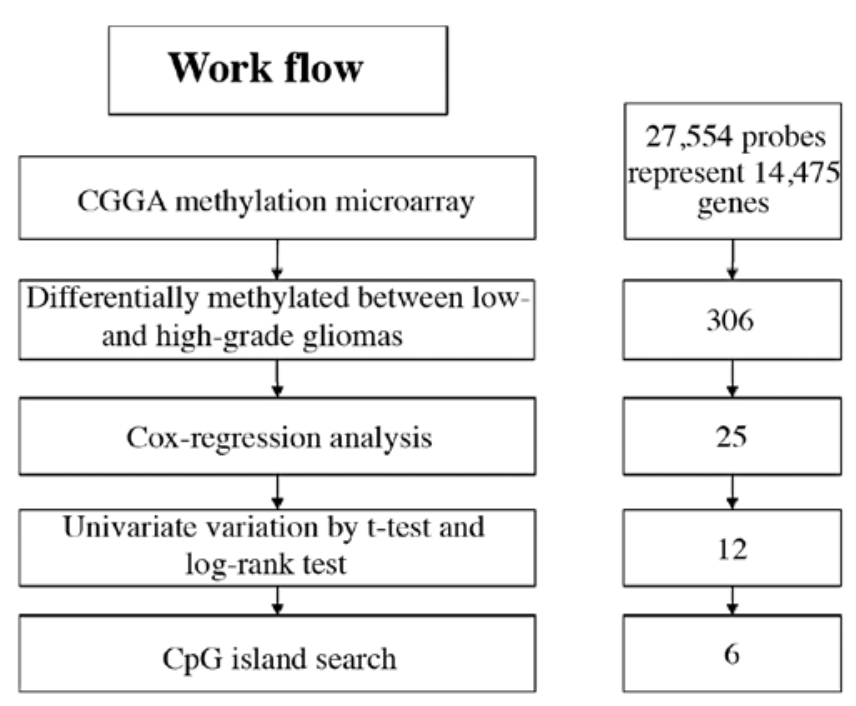

Figure 1. Work flow. CGGA methylation microarray, differently methylated between high- and low-grade gliomas, Cox-regression analysis, univariate validation by t-test and log-rank test and $\mathrm{CpG}$ island search were used to screen out the gene of interested. In the end, there were 6 genes in the list.

invasion and colony formation number between treated and control groups were analyzed by t-test. $\mathrm{P}<0.05$ was considered to indicate a statistically significant result.

\section{Results}

Gene screening. The methylation microarray from CGGA contains 27,578 highly informative $\mathrm{CpG}$ sites covering more than 14,475 human RefSeq genes. Following SAM analysis between high- and low-grade gliomas, with FDR $<0.2,306$ probes were screened out. Then, by survival Cox-regression analysis using Metlab software, 25 probes (25 genes) were left. For univariate analysis validation, we performed t-test and Kaplan-Meier survival curve and log-rank test, after which, 12 probes (12 genes) remained. We further searched for the CpG islands upstream of the promoter, 6 genes were in the list in the end. These 6 genes were ADCY1, KAZALD1, KLF4, SLMAP, TETRAN and TP53INP1. We selected the oncogene KAZALD1 (Fig. 1).

High grade and poor prognosis association of KAZALDI hypomethylation in CGGA data. The methylation level of KAZALD1 was measured in a series of 119 glioma samples (63 low-grade gliomas, 33 anaplastic gliomas and 23 glioblastomas) via microarray. KAZALD1 was significantly hypomethylated in high-grade gliomas compared to low-grade gliomas $(\mathrm{P}<0.001)$. The correlation between KAZALD1 methylation and OS was measured through Kaplan-Meier survival curve analysis and log-rank test. KAZALD1 methylation was inversely correlated with $\mathrm{OS}$ in the high-grade glioma samples $(\mathrm{P}=0.002)$ (Fig. 2B).

Patients with low-methylation level had a median OS of 544 days compared to 958 days in patients with high methylation level (Table I). Preoperative KPS score was also correlated with OS $(\mathrm{P}=0.004)$. There was no significant association 
Table I. Variables related to OS in 56 high-grade gliomas in methylation microarray: univariate and multivariate analysis.

\begin{tabular}{|c|c|c|c|c|c|c|c|}
\hline \multirow[b]{2}{*}{ Variables } & \multirow[b]{2}{*}{$\begin{array}{c}\text { No. of } \\
\text { patients }\end{array}$} & \multicolumn{3}{|c|}{ Univariate analysis } & \multicolumn{3}{|c|}{ Multivariate analysis } \\
\hline & & $\begin{array}{l}\text { Median OS } \\
\quad \text { (days) }\end{array}$ & $\begin{array}{l}95 \% \text { CI } \\
\text { (days) }\end{array}$ & P-value & $\begin{array}{l}\text { Relative } \\
\text { risk }\end{array}$ & $95 \% \mathrm{CI}$ & P-value \\
\hline \multicolumn{8}{|l|}{ Gender } \\
\hline Male & 23 & 591 & $249-745$ & & & & \\
\hline Female & 33 & 497 & $317-865$ & 0.528 & & & \\
\hline \multicolumn{8}{|c|}{ Age (years) } \\
\hline$\leq 41.5$ & 28 & 878 & $304-1452$ & & & & \\
\hline$>41.5$ & 28 & 530 & $293-767$ & 0.178 & 1.248 & $0.605-2.573$ & 0.549 \\
\hline \multicolumn{8}{|l|}{ KPS } \\
\hline$<80$ & 19 & 290 & $135-445$ & & & & \\
\hline$\geq 80$ & 37 & 878 & NR & 0.004 & 0.426 & $0.208-0.871$ & 0.019 \\
\hline \multicolumn{8}{|c|}{ KAZALD1-methylation } \\
\hline Low & 28 & 544 & $400-688$ & & & & \\
\hline High & 28 & 958 & $768-1150$ & 0.002 & 0.367 & $0.172-0.782$ & 0.009 \\
\hline
\end{tabular}

NR, not reached; CI, confidence interval; KPS, Karnofsky performance status.

A

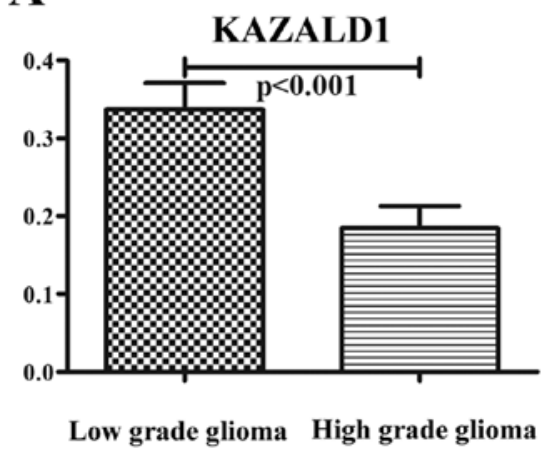

B

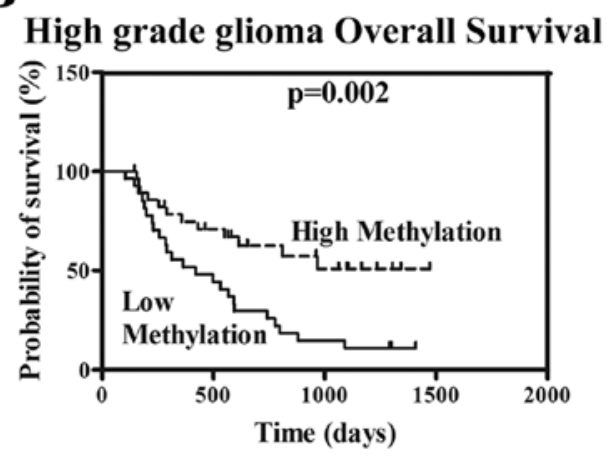

Figure 2. High grade and poor prognosis association of KAZALD1 hypomethylation in CGGA data. (A) The methylation level of KAZALD1 promoter was significantly lower in high-grade glioma patients than those in low-grade $(\mathrm{P}<0.001)$. (B) In high-grade gliomas, patients with low methylation level of KAZALD1 had shorter OS than those with high methylation $(\mathrm{P}=0.002)$.

with age, gender and OS. The multivariate Cox proportional hazards model, after adjusting for age, KPS score, identified hypomethylation of KAZALD1 as an independent unfavorable prognostic factor for OS $(\mathrm{P}=0.009)$.

Expression of KAZALD1 is relative to glioma grade progression and confers a poor prognosis of high KAZALDI expression in high-grade glioma patients. We performed immunohistochemical staining in 91 glioma samples from mainland Han Chinese glioma patients. The 91 patients consisted of 30 patients with astrocytoma (A, WHO grade II), 20 with anaplastic astrocytoma (AA, WHO grade III) and 41 with glioblastoma multiforme (GBM, WHO grade IV). We analyzed the correlation between KAZALD1 protein expression and histological staging of gliomas, which showed KAZALD1 expression ranged from low to high along with grade progression of gliomas $(\mathrm{P}<0.01$; Fig. $3 \mathrm{~A})$. Survival analysis showed that patients with high KAZALD1 expression had significantly shorter OS $(\mathrm{P}<0.001)$ than those with low expression in high-grade glioma patients (Fig. 3B). We also confirmed there was no KAZALD1 expression in normal brain tissue by IHC (Fig. 4).

Patients with high KAZALD1 expression had median OS time of 295 days compared to 660 days in patients with low KAZALD1 expression (Table II). Preoperative KPS score was also correlated with $\mathrm{OS}(\mathrm{P}=0.022)$. There were no significant associations with age, gender and OS. The multivariate Cox proportional hazards model, after adjusting for age, KPS score, identified high KAZALD1 expression as an independent unfavorable prognostic factor for OS (HR, 4.096; $\mathrm{P}=0.001)$.

KAZALD1 promotes invasion and proliferation of LN229 and U251 cell lines. Following siRNA knockdown of KAZALD1 in two cell lines, LN229 and U251, we validated its expres- 
Table II. Variables related to OS in 61 high-grade glioma validated samples (58 survivals were available): univariate and multivariate analysis.

\begin{tabular}{|c|c|c|c|c|c|c|c|}
\hline \multirow[b]{2}{*}{ Variables } & \multirow[b]{2}{*}{$\begin{array}{l}\text { No. of } \\
\text { patients }\end{array}$} & \multicolumn{3}{|c|}{ Univariate analysis } & \multicolumn{3}{|c|}{ Multivariate analysis } \\
\hline & & $\begin{array}{l}\text { Median } \\
\text { OS (days) }\end{array}$ & $\begin{array}{c}95 \% \text { CI } \\
\text { (days) }\end{array}$ & P-value & $\begin{array}{l}\text { Relative } \\
\text { risk }\end{array}$ & $95 \% \mathrm{CI}$ & P-value \\
\hline \multicolumn{8}{|l|}{ Gender } \\
\hline Male & 38 & 493 & $339-647$ & & & & \\
\hline Female & 20 & 362 & $200-523$ & 0.410 & & & \\
\hline \multicolumn{8}{|c|}{ Age (years) } \\
\hline$\leq 50$ & 29 & 570 & $466-674$ & & & & \\
\hline$>50$ & 29 & 354 & $332-376$ & 0.192 & 1.207 & $0.541-2.689$ & 0.646 \\
\hline \multicolumn{8}{|l|}{ KPS } \\
\hline$<80$ & 37 & 376 & $245-507$ & & & & \\
\hline$\geq 80$ & 21 & 572 & $461-683$ & 0.022 & 0.519 & $0.207-1.300$ & 0.161 \\
\hline \multicolumn{8}{|c|}{ KAZALD1-expression } \\
\hline Low & 36 & 660 & $467-853$ & & & & \\
\hline High & 22 & 295 & $184-406$ & 0.000 & 4.096 & $1.843-9.106$ & 0.001 \\
\hline
\end{tabular}

A

KAZALD1 expression

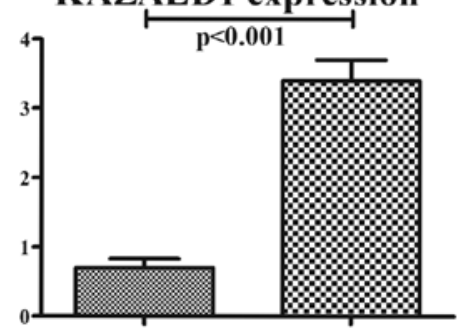

Low grade glioma High grade glioma
B

High grade glioma Overall Survival

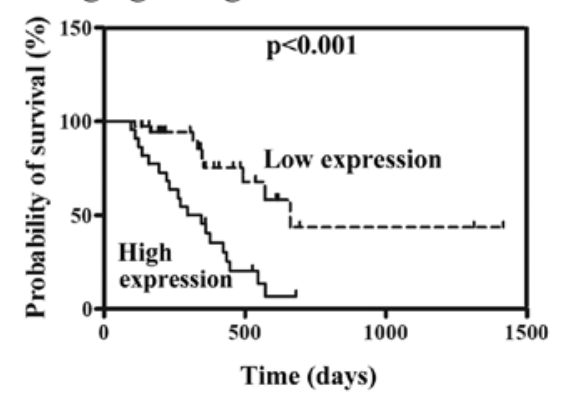

Figure 3. KAZALD1 expression correlates with glioma grade progression and prognosis. (A) The expression of KAZALD1 was significantly higher in high-grade glioma patients than in those with low-grade glioma $(\mathrm{P}<0.001)$. (B) High KAZALD1 expression had significantly shorter $\mathrm{OS}(\mathrm{P}<0.001)$ than low expression in high-grade glioma patients.

sion level (Fig. 5A). The cells were further used for transwell invasion assay and colony formation assay which showed that siRNA significantly attenuated the effect of KAZALD1 on cell invasion $(\mathrm{P}<0.05$; Fig. $5 \mathrm{~B})$ as well as proliferation $(\mathrm{P}<0.05$; Fig. 5C), respectively.

KAZALD1 promotes glioma growth in vivo. To investigate the potential impact of KAZALD1 expression in vivo, a U251 xenograft model was utilized. The KAZALD1-treated group displayed a significant growth and tumor reduction, whereas tumor growth was not impacted by negative control (Fig. 6C).

\section{Discussion}

In the present study, we investigated the methylation and expression level of KAZALD1 in two independent cohorts, totaling 210 glioma patients. We found that methylation level of KAZALD1 promoter was associated with glioma grade progression and overall survival (OS) in high-grade gliomas using genome-wide DNA methylation profiling. We further compared the expression level of KAZALD1 in low-grade (astrocytoma, oligodendrocytoma and oligoastrocytoma) and high-grade (anaplastic gliomas and glioblastomas) human gliomas, and demonstrated a significant increase in KAZALD1 expression from low- to high-grade gliomas. KAZALD1 was an independent prognostic factor predicting OS in highgrade gliomas, indicating a significant correlation between KAZALD1 expression and clinical outcome in glioma patients. KAZALD1 functional analyses were performed in LN229 and U251 cell lines, which showed KAZALD1 promoted invasion and proliferation of glioma both in vitro and in vivo. To the best of our knowledge, this is the first report on the methylation status, expression difference and function of KAZALD1 in glioma. 


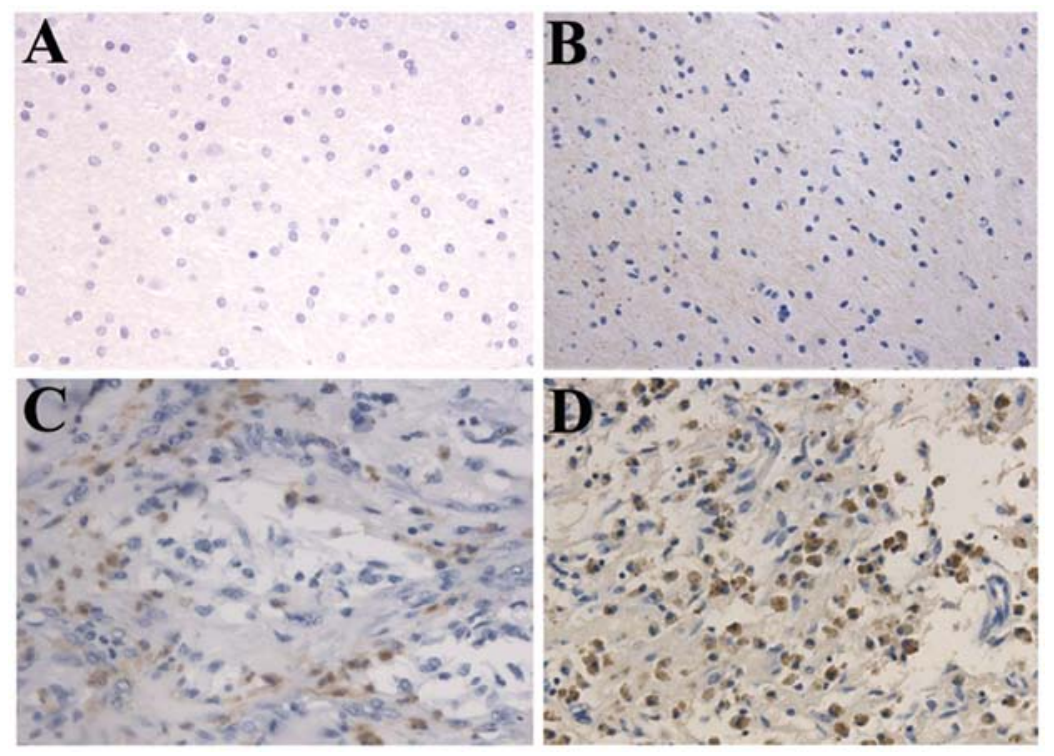

Figure 4. KAZALD1 expression correlates with glioma grade progression and prognosis. Immunohistochemical staining showed KAZALD1 expression ranged from low to high along with grade progression of gliomas. (A) Normal brain tissue, (B) astrocytoma (WHO grade II), (C) anaplastic astrocytoma (WHO grade III) and (D) glioblastoma(WHO grade IV).

A

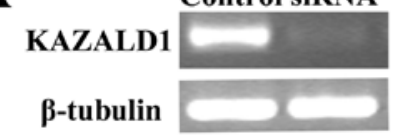

B
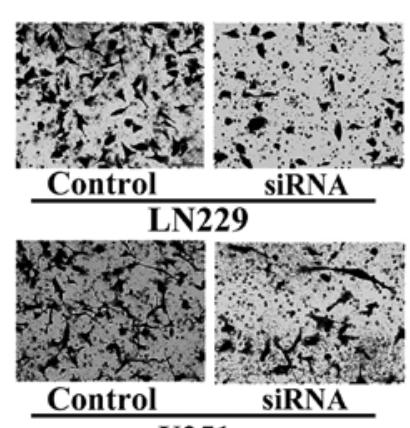

U251

C

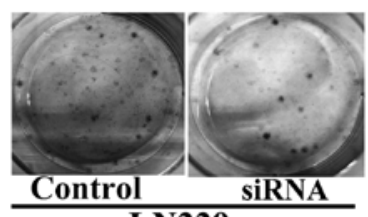

LN229

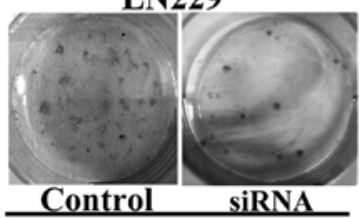

U251
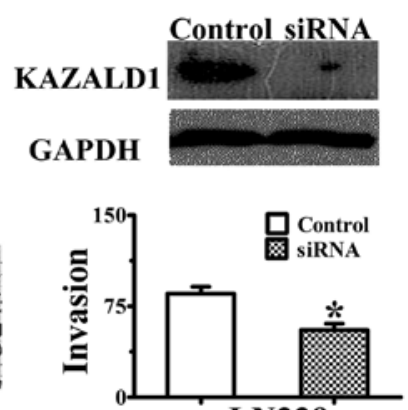

LN229

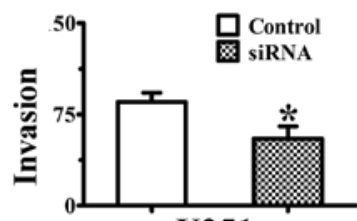

U251

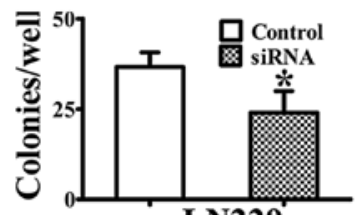

LN229

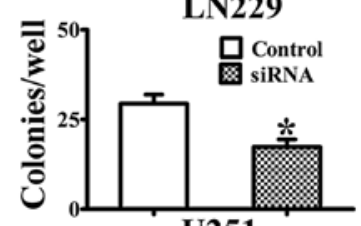

U251

Figure 5. KAZALD1 promotes invasion and proliferation of LN229 and U251 cell lines. (A) KAZALD1 expression was significantly knocked down by siRNA showing in RNA level $(\mathrm{P}<0.01)$ and protein level (western blot identified, $\mathrm{P}<0.01$ ). (B) Invasion of LN229 and U251 cell lines assessed by transwell assay. The number of invading cells was significantly less in the siRNA group compared to the control group. Number of invading cells shown as a histogram. ${ }^{*} \mathrm{P}<0.05$ vs. control. (C) The number of colonies formed by cells treated with siRNA of KAZALD1 was decreased compared to that of control cells. Number of colonies shown as a histogram." $\mathrm{P}<0.05$ vs. control.

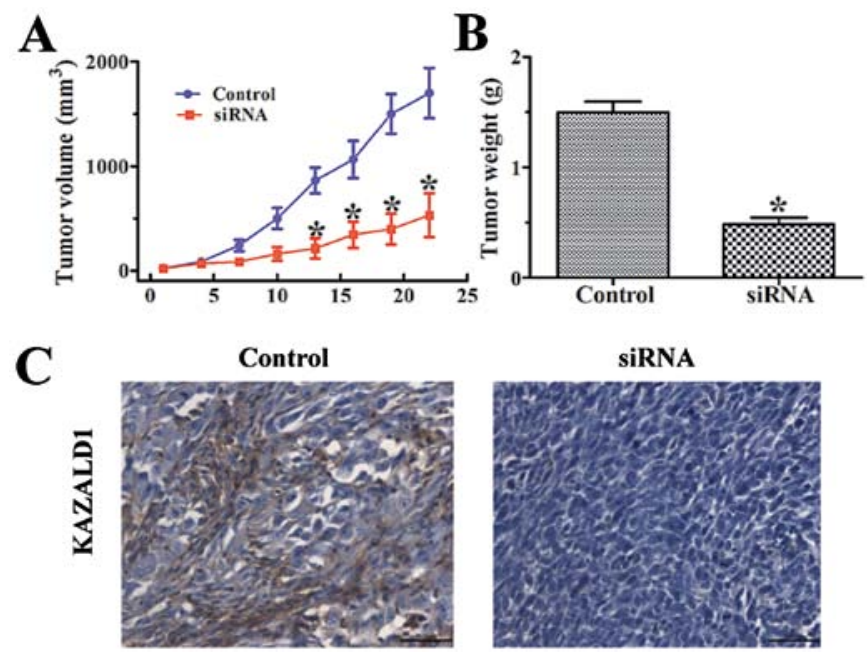

Figure 6. KAZALD1 promotes glioma growth in vivo. (A) U251 tumors were treated by siRNA of KAZALD1 and negative control. Growth curves for siRNA of KAZALD1-treated U251 tumors $(n=6)$ vs. negative control group $(n=6)$. (B) Tumors were resected and tumor mass was determined 22 days following implantation. Data indicate decreased tumor mass siRNA KAZALD1-treated U251 tumors compared with the negative control group. (C) IHC assay for KAZALD1 expression in negative control-treated U251 tumors vs. siRNA of KAZALD1 xenograft. ${ }^{*} \mathrm{P}<0.05$.

DNA methylation of promoter $\mathrm{CpG}$ islands has been recognized as an important mechanism for the regulation of gene expression and transcriptional modification in mammals. DNA methylation status is more stable and can be detected using a number of high-throughput and sensitive techniques with little patient material (21). Aberrations in DNA methylation patterns may have critical effects on tumor initiation and progression. Although considerable research has been conducted on the epigenetic control of tumor suppressor genes, little is known about the potential role of promoter $\mathrm{CpG}$ demethylation in the activation of oncogenes. In the 
present study, we performed genome-wide DNA methylation profiling of 119 glioma tissues via microarrays. We set a series of screening conditions including grade correlation, survival correlation and $\mathrm{CpG}$ island in upstream of gene promoter (Fig. 1). KAZALD1 was one of six genes conformed to appeal conditions. We also found that methylation level of KAZALD1 in high-grade glioma was significantly lower than that in low-grade glioma (Fig. 2A). Kaplan-Meier survival curve analysis showed that low methylation level of KAZALD1 had shorter OS compared to the high methylation group in high-grade glioma patients (Fig. 2B). Multivariate analysis identified hypomethylation of KAZALD1 was an independent unfavorable prognostic factor for OS (Table I; $\mathrm{P}=0.009$; low methylated group, 544 days; high methylated group, 958 days).

To explore whether the expression level of KAZALD1 correlated with the status of methylation in glioma, we detected the KAZALD1 expression in 91 independent cohorts including 30 low-grade and 61 high-grade glioma patients using immunohistochemistry. We found that high-grade glioma patients had significantly higher KAZALD1 expression than low-grade glioma patients (Figs. 3A and 4). According to our original hypothesis, there was a negative correlation between KAZALD1 expression and methylation status in low- and high-grade gliomas. In 61 high-grade glioma patients, patients with high KAZALD1 expression had shorter OS than those with low expression (Fig. 3B, Table II). These observations indicated that glioma with KAZALD1 hypomethylation and higher protein expression might be a more biologically aggressive phenotype than those without it. These results showed that increased expression of KAZALD1 in gliomas with a poor prognosis was at least partially due to hypomethylation of the KAZALD1 promoter region.

To date, no information has been provided regarding the regulation of the KAZALD1 gene in gliomas. However, rather limited data are available on the relationship between the KAZALD1 gene and its precise mechanism of action on tumors, and their clinical effect on outcome for patients with glioma remains unclear. The function of KAZALD1 in dental development and bone regeneration was investigated only recently and, thus far, its biological function is unknown (17). The KAZALD1 insulin-like factor binding domain has high affinity for insulin-like growth factors, while the Kazal domain contains sequence and structural homology to serine proteases and follistatins (15). The immunoglobulin-like domain appears to have a molecular binding function perhaps regulating cell adhesion and extracellular ligand binding (16), and is associated with regions of matrix mineralization (17). KAZALD1 and MMP-20 are coexpressed in secretory odontoblasts at developmental stages (22). Based on the above findings, we hypothesize that KAZALD1 promoted the glioma progression through invasion and proliferation. We performed functional assays in LN229 and U251 cell lines and found that invasion and colony formation number decreased when KAZALD1 was knocked down by siRNA, and the same results were founded in vivo. We consider that KAZALD1 could combine with IGF, to activate the IGF pathway. A series of downstream genes of the IGF pathway promote glioma grade progression and shorten survival time. However, this requires further assays.
In summary, promoter hypomethylation of KAZALD1 was associated with high KAZALD1 expression. Patients harboring hypomethylation and high expression KAZALD1 had shorter OS in high-grade glioma. On the basis of these observations and the results from subset analysis, it is reasonable to conclude that KAZALD1 promoter methylation status is an important prognostic biomarker in glioma. KAZALD1 promoted glioma malignant progression through invasion and proliferation.

\section{Acknowledgements}

The authors thank Dr Susan Furness for her critical reading and Professor Chen (Beijing Sanbo Brain Hospital) for IHC technical support. This study was supported by grants from the National High Technology Research and Development Program (no. 2012AA02A508), the International Science and Technology Cooperation Program (no. 2012DFA30470), the National 973 program (no. 2011CB707804) and the National Natural Science Foundation of China (no. 81201993).

\section{References}

1. Zhang W, Yan W, You G, Bao Z, Wang Y, et al: Genome-wide DNA methylation profiling identifies ALDH1A3 promoter methylation as a prognostic predictor in G-CIMP-primary glioblastoma. Cancer Lett 328: 120-125, 2013.

2. Zhang J, Huang K, Shi Z, Zou J, Wang Y, et al: High $\beta$-catenin/Tcf-4 activity confers glioma progression via direct regulation of AKT2 gene expression. Neuro Oncol 13: 600-609, 2011.

3. Chen L, Han L, Zhang K, Shi Z, Zhang J, et al: VHL regulates the effects of miR-23b on glioma survival and invasion via suppression of HIF- $1 \alpha /$ VEGF and $\beta$-catenin/Tcf- 4 signaling. Neuro Oncol 14: 1026-1036, 2012.

4. Yan W, Zhang W, You G, Zhang J, Han L, et al: Molecular classification of gliomas based on whole genome gene expression: a systematic report of 225 samples from the Chinese Glioma Cooperative Group. Neuro Oncol 14: 1432-1440, 2012.

5. Wang Y, Li S, Chen L, You G, Bao Z, et al: Glioblastoma with an oligodendroglioma component: distinct clinical behavior, genetic alterations, and outcome. Neuro Oncol 14: 518-525, 2012.

6. Zhang W, Zhang J, Yan W, You G, Bao Z, et al: Whole-genome microRNA expression profiling identifies a 5-microRNA signature as a prognostic biomarker in Chinese patients with primary glioblastoma multiforme. Cancer 119: 814-824, 2013.

7. Zhang W, Zhang J, Hoadley K, Kushwaha D, Ramakrishnan V, et al: miR-181d: a predictive glioblastoma biomarker that downregulates MGMT expression. Neuro Oncol 14: 712-719, 2012.

8. Starkweather AR, Sherwood P, Lyon DE, McCain NL, Bovbjerg DH, et al: A biobehavioral perspective on depressive symptoms in patients with cerebral astrocytoma. J Neurosci Nurs 43: 17-28, 2011

9. Jones PA and Baylin SB: The epigenomics of cancer. Cell 128: 683-692, 2007.

10. Gaspar-Maia A, Alajem A, Meshorer E and Ramalho-Santos M: Open chromatin in pluripotency and reprogramming. Nat Rev Mol Cell Biol 12: 36-47, 2011.

11. Feinberg AP and Tycko B: The history of cancer epigenetics. Nat Rev Cancer 4: 143-153, 2004.

12. Baylin SB: DNA methylation and gene silencing in cancer. Nat Clin Pract Oncol 2 (Suppl 1): S4-S11, 2005.

13. Ehrlich M: DNA methylation in cancer: too much, but also too little. Oncogene 21: 5400-5413, 2002.

14. Cadieux B, Ching TT, VandenBerg SR and Costello JF: Genomewide hypomethylation in human glioblastomas associated with specific copy number alteration, methylenetetrahydrofolate reductase allele status, and increased proliferation. Cancer Res 66: 8469-8476, 2006. 
15. Forbes B, Szabo L, Baxter RC, Ballard FJ and Wallace JC: Classification of the insulin-like growth factor binding proteins into three distinct categories according to their binding specificities. Biochem Biophys Res Commun 157: 196-202, 1988.

16. Bork P, Holm L and Sander C: The immunoglobulin fold Structural classification, sequence patterns and common core. J Mol Biol 242: 309-320, 1994.

17. James MJ, Jarvinen E and Thesleff I: Bonol: a gene associated with regions of deposition of bone and dentine. Gene Expr Patterns 4: 595-599, 2004.

18. Li S, Yan C, Huang L, Qiu X, Wang Z, et al: Molecular prognostic factors of anaplastic oligodendroglial tumors and its relationship: a single institutional review of 77 patients from China. Neuro Oncol 14: 109-116, 2012.

19. Hill VK, Ricketts C, Bieche I, Vacher S, Gentle D, et al: Genomewide DNA methylation profiling of $\mathrm{CpG}$ islands in breast cancer identifies novel genes associated with tumorigenicity. Cancer Res 71: 2988-2999, 2011.
20. Zhang W, Qiu XG, Chen BS, Li SW, Cui Y, et al: Antiangiogenic therapy with bevacizumab in recurrent malignant gliomas: analysis of the response and core pathway aberrations. Chin Med J 122: 1250-1254, 2009 .

21. Schatz P, Distler J, Berlin K and Schuster M: Novel method for high throughput DNA methylation marker evaluation using PNA-probe library hybridization and MALDI-TOF detection. Nucleic Acids Res 34: e59, 2006.

22. Kiukkonen A, Sahlberg C, Lukinmaa PL, Alaluusua S, Peltonen E, et al: 2,3,7,8-tetrachlorodibenzo-p-dioxin specifically reduces mRNA for the mineralization-related dentin sialophosphoprotein in cultured mouse embryonic molar teeth. Toxicol Appl Pharmacol 216: 399-406, 2006. 